\title{
Application of interferometric wave propagation for refractive index measurements of solids
}

\author{
Glen D. Gillen \\ Air Force Research Laboratory, Materials and Manufacuring Directorate/MLPJ, Anteon Corporation, \\ Building 71A, Area B, Wright-Patterson Air Force Base, Dayton, OH 45433 \\ Voice: 937 252-3132 ext.3039,Fax: 937 252-0418,Email: glen.gillen@wpafb.af.mil \\ Shekhar Guha \\ Air Force Research Laboratory, Materials and Manufacturing Directorate, Wright-Patterson AFB, OH 45433-7702
}

\begin{abstract}
The output signal of a modified Michelson interferometer with a flat-parallel sample, whose normal is oriented at an arbitrary angle with respect to the laser path, placed in one arm is analyzed. The interferometric pattern as a function of angle is calculated, and used to determine the absolute refractive index.

(C)2003 Optical Society of America

OCIS codes: (260.3160) Physical Optics, Interference, (260.3060) Physical Optics, Infrared
\end{abstract}

\section{Introduction}

Although small changes in the refractive index of a material can be accurately measured using interferometry, this technique is usually not used for measuring the absolute values of the refractive indicies. In this work, we present a detailed theoretical treatment based on physical optics to show how the absolute value of the refractive index of a material can be determined using interferometry

\section{Method}

If a sample is placed in one arm of a Michelson interferometer and rotated about an axis perpendicular to the incident laser path the phase of the laser field for that arm becomes a function of the sample orientation angle and will interfere with the laser field that passes through the other arm of the interferometer.

Treating the laser fields as complex electromagnetic waves and using wave propagation techniques, the observed interference pattern can be expressed as

$$
\text { Signal } \propto \cos \left[\frac{4 \pi d}{\lambda}\left(\sqrt{n^{2}-\sin ^{2} \theta}+1-\cos \theta\right)+\phi\right]
$$

where $d$ is the wafer thickness, $\lambda$ is the laser wavelength, $n$ is the real part of the refractive index, $\theta$ is the angle between the incident laser path and the sample normal, and $\phi$ is the phase difference between the reference arm and the sample arm at normal incidence.

The simple design of this method allows itself to easily be adapted, experimentally, for refractive index measurements to be conducted for samples at cryogenic temperatures as well.

\section{Discussion}

Our talk will discuss in detail the assumptions and mathematical treatment of the laser field for each arm of the interferometer that lead to equation (1), using wave propagation techniques. We will also present experimental data on several infrared materials at both room temperature and cryogenic temperatures measured using a grating tuned carbon dioxide laser at $10.6 \mu \mathrm{m}$ and provide a comparison of the experimental data with theoretical predictions. 